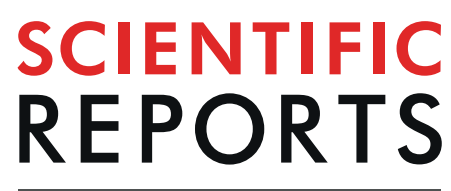

natureresearch

\title{
Detection and identification of transgenic events by next generation sequencing combined with enrichment technologies
}

\author{
Frédéric Debode ${ }^{1,4^{*}}$, Julie Hulin $\mathbb{1}^{1,4}$, Benoît Charloteaux ${ }^{2}$, Wouter Coppieters ${ }^{2}$, \\ Marc Hanikenne $^{3}$, Latifa Karim ${ }^{2}$ \& Gilbert Berben ${ }^{1}$
}

Next generation sequencing (NGS) is a promising tool for analysing the quality and safety of food and feed products. The detection and identification of genetically modified organisms (GMOs) is complex, as the diversity of transgenic events and types of structural elements introduced in plants continue to increase. In this paper, we show how a strategy that combines enrichment technologies with NGS can be used to detect a large panel of structural elements and partially or completely reconstruct the new sequence inserted into the plant genome in a single analysis, even at low GMO percentages. The strategy of enriching sequences of interest makes the approach applicable even to mixed products, which was not possible before due to insufficient coverage of the different genomes present. This approach is also the first step towards a more complete characterisation of agrifood products in a single analysis.

The number and diversity of GMOs have greatly increased in recent years. Currently, the reference method for GMO detection is real-time PCR. The main problem of real-time PCR is that it can only be used to detect targeted sequences, which means that searches are somewhat limited since they can only find what is being looked for. Moreover, new solutions need to be found for the characterisation of authorised and unauthorised GMOs. NGS approaches may address the problem of identifying all GMOs in a sample. High-throughput sequencing can sequence several million fragments in parallel and is able to provide the whole sequence of plant genomes ${ }^{1}$. NGS has already been used to help molecularly characterise a genetically modified (GM) soybean without the need for Southern Blot analysis ${ }^{2}$. Several approaches have been developed that use the potential of high-throughput sequencing for the detection of GMOs or GMO-derived products ${ }^{3-6}$. However, NGS is still not frequently used for GMO detection due to important challenges, such as uneven coverage of the genome ${ }^{7}$. This problem can be reinforced as a function of the genome size of the plant considered, e.g., the soybean genome is $\sim 1.1$ gigabases $(\mathrm{Gb})^{8}$ while the wheat genome is $\sim 17 \mathrm{~Gb}^{9}$, and genetic diversity is even greater in complex food products containing several plant species.

Several approaches for GMO detection have already been developed. First, pilot studies have shown that NGS using whole genome sequencing approaches is able to detect GMOs ${ }^{10-12}$. NGS became a method for checking for inserted sequences ${ }^{2-4}$. However, these methods have only been tested on pure GM material, while a large number of sequencing runs would be required to gain sufficient coverage to allow the detection of low GM contents ${ }^{7}$. To detect GMOs present at low levels, sequencing of a large number of targeted amplicons by NGS was proposed $^{13}$. This method was able to detect numerous structural elements but was not suitable for reconstructing the inserted sequence. The sensitivity of this method was not evaluated in depth, but its performance was poorer than real-time PCR ${ }^{13}$. Only techniques combining NGS with SiteFinding PCR ${ }^{5}$ and DNA walking strategies ${ }^{14,15}$ have been able to provide information on the junction sequence between a plant and GM construct at low percentages. The method using genome walking with ALF (amplification of linearly enriched fragment) could detect a level

${ }^{1}$ Walloon Agricultural Research Center (CRA-W), Unit Traceability and Authentication, chaussée de Namur 24, 5030, Gembloux, Belgium. 'University of Liège, GIGA - Genomics Platform, B34, 4000, Liège (Sart Tilman), Belgium. ${ }^{3}$ University of Liège, InBioS - PhytoSystems, Functional Genomics and Plant Molecular Imaging, Chemin de la Vallée, 4, B22, 4000, Liège (Sart Tilman), Belgium. ${ }^{4}$ These authors contributed equally: Frédéric Debode and Julie Hulin. *email: f.debode@cra.wallonie.be 
as low as $1 \%{ }^{15}$. This method starts with two structural elements, p35S and tNOS. DNA walking method using anchored PCR followed by two semi-nested PCRs was able to detect a level of $0.1 \%$ of Bt rice ${ }^{14}$. This method is now capable of starting from five structural elements (p35S, t35S pCambia, tNOS and cry) ${ }^{14,16}$. However, these strategies, based on the sequencing of amplicons by NGS, are time-consuming, cannot cover large fragments of GM constructs and are dependent on a starting point linked to the presence of a precise structural element.

We developed an approach combining NGS with a strategy of enriching the regions of interest that differs from the eleven enrichment strategies listed by Arulandhu et al. ${ }^{17}$. The regions of interest correspond to a series of structural elements frequently introduced into transgenic constructs. We checked the capacity of the method for GMO detection, even in flour containing low percentages of transgenic plants, and developed a bioinformatic pipeline for the detection and characterisation of GM events.

\section{Results and Discussion}

Our work started with the development of a database of sequences that could be used for enrichment. The present version of the database gathers the sequences of 10 promoters, 6 terminators and 23 genes or miscellaneous elements that are found in transgenic constructs (Table 1). The total size of the enrichment sequences in the database is $\sim 53 \mathrm{~kb}$, but the database is still far from its limit as the methodology can be scaled up to $24 \mathrm{Mb}$. The covering a large number of GM events is possible, as the database includes the sequences of the structural elements most commonly used in genetically modified plants ${ }^{18,19}$. Sequences corresponding to antibiotic resistance or other selection markers were not included in the database, as they could generate unexpected signals linked to the presence of traces of DNA from the bacteria and recombinant plasmids used for the production of the enzymes employed for PCR amplification and sequencing. If we compare the potential of detection with the $328 \mathrm{GM}$ events listed in the GMOseek matrix ${ }^{19}$ and in relation to 23 plant species, only 3 GM events (AR9 Azuki bean, LY038 maize and BPS-CV127-9 soybean) would not be detected because they do not contain any of the 40 structural elements used to design the enrichment. AR9 Azuki bean, LY038 maize and BPS-CV127-9 soybean contain structural elements that are particular to these transgenic events. The sequences of these structural elements are not currently available but could be added in the future. However, the AR9 Azuki bean also contains nptII, providing tolerance to antibiotics ${ }^{20}$. This example shows the importance of not excluding selection markers from the enrichment database in the future and is why the pros and cons of the presence of such sequences should be evaluated in the next version of the enrichment database.

The developed database was then used to create capture probes focusing on the elements listed in Table 1. Two types of methodologies were tested for sequence enrichment through capture probes. The first methodology used numerous probes of 50-80 bp that had a high level of overlap (SeqCapEZ technology, Roche Diagnostics/ NimbleGen, Madison, WI), in which each base is generally covered by at least 7 probes. The second methodology used larger probes ( 120 bp) with a low level of overlap (SureSelect technology, Agilent Technologies, Santa Clara, CA). No degeneracy was introduced in the probes. The probes are supposed to be able to catch fragments of up to $500 \mathrm{bp}$ in size, which would allow the captured fragments to include junctions between structural elements or junctions between the plant and inserted sequence.

The enrichment principle is presented in Fig. 1. From a theoretical point of view, both methodologies have advantages: shorter and more numerous probes should be better at capturing degraded DNA or sequences of structural elements that slightly vary from what is expected, while longer probes should lead to increased specificity of sequence capture. The comparison of the SureSelect and NimbleGen technologies has already been discussed for several medical applications with results favouring either the NimbleGen approach ${ }^{21-23}$ or SureSelect technology $\mathrm{y}^{24}$ or indicating comparable performances ${ }^{25}$. The comparisons show that both methodologies have pros and cons depending on the objectives of the project ${ }^{26}$ and indicate that the balance in favour of one method can change as a function of the evolution of kits and protocols ${ }^{26}$.

In this study, after analysing sequencing runs on Illumina devices, better enrichments with fewer unexpected assignations were observed when using SureSelect technology. This paper focuses on the best results obtained with this technology. After enrichment, the DNA libraries were sequenced on an Illumina MiSeq system (Illumina, San Diego, CA).

To analyse the large amount of read data, a bioinformatic workflow was created. The workflow was divided into two parts. In the first part, which was aimed at GMO detection, reads were aligned onto the sequences used for enrichment and filtered according to their alignment scores. Statistical analysis was then performed to determine whether the reads could be distinguished from noise and assimilated to positive results. The objective of the second part of the workflow was to characterise the GMO through the creation of contigs in an attempt to reconstruct the whole transgene, possibly including the plant-construct junction specific to the event. The bioinformatics workflow used different scripts and programs, as presented in Fig. 2.

The analysed samples included five species, eight transgenic events and variable fractions $(0.1 \%, 1 \%, 10 \%$ and $100 \%$ ) of GMOs (Table 2 in the methods section). Concerning GMO detection, the structural elements listed in the enrichment database and present in the GM events tested were all detected (Figs 3 and 4).

Logically, the percentage of sequenced reads assigned to the structural element present depended on the GM percentage. An example is given in Table 3 for GTS-40-3-2 soybean, in which the percentage of reads aligned with p35S, tNOS and EPSPS increased as a function of the GM percentage. The absolute number of reads is linked to the length of the structural elements (this point can, however, be normalised) and to the DNA quantities introduced in the experiments. The number of reads cannot be used for quantitative approaches, and sequencing will not replace real-time PCR or digital PCR for GMO quantification. However, once the system is updated with taxon-specific genes (preliminary experiments are underway), the system may be able to provide an indication of the GM percentage. This information would, however, remain semi-quantitative.

In the bioinformatics workflow, a threshold level was set for considering an element beyond background noise and thus as being detected. In the SureSelect experiments, this threshold was based on the mean number 


\begin{tabular}{|c|c|c|c|}
\hline Type of structural element & Name & Size (bp) & Sequence source \\
\hline \multirow{11}{*}{ Promoters } & $\mathrm{p} 35 \mathrm{~S}$ & 867 & NCBI KX880509 \\
\hline & pFMV & 981 & NCBI X06166 \\
\hline & pUbi & 2018 & NCBI S94464 \\
\hline & pNOS & 398 & Patent WO2006074956 \\
\hline & pmas & 660 & NCBI DQ225747 \\
\hline & Ps7s7 & 1046 & NCBI AY181091 \\
\hline & pRice actin 1 & 660 & NCBI S44221 \\
\hline & pRice actin2 & 259 & NCBI EU161577 \\
\hline & pSSuAra & 1727 & NCBI CP002684 \\
\hline & pTA29 & 627 & NCBI X52283 \\
\hline & pMTL & 2556 & NCBI S57628 \\
\hline \multirow{6}{*}{ Terminators } & tOCS & 823 & NCBI LT727071 \\
\hline & tE9 & 648 & Patent W02007027777 \\
\hline & tNOS & 300 & NCBI AB809952 \\
\hline & $\operatorname{tg} 7$ & 203 & Patent WO2006074956 \\
\hline & tpinII & 318 & NCBI KP784700 \\
\hline & t35S & 211 & NCBI GU734649 \\
\hline \multirow{22}{*}{ Genes } & gus & 576 & NCBI CP029981 \\
\hline & gox & 1296 & Patent US5463175 \\
\hline & cryIAb 1 & 1854 & Patent US20030226171 \\
\hline & cryIAb 2 & 3844 & NCBI AY326434 \\
\hline & cryIAb 3 & 1947 & Patent US5625136 \\
\hline & crylB & 1950 & NCBI KC414884 \\
\hline & crylAa & 1848 & NCBI GU583855 \\
\hline & cryl Ab/c & 1923 & NCBI GU583854 \\
\hline & crylAc & 1923 & NCBI KF630361 \\
\hline & crylA105 1 & 3537 & Patent WO200702777 \\
\hline & crylA105 2 & 3433 & NCBI DI362404 \\
\hline & cry2Ab2 & 1900 & NCBI DI362404 \\
\hline & cry3A055 & 1797 & Patent EP2289311 \\
\hline & cry34Ab1 & 424 & Patent W02006039376 \\
\hline & cry35Ab1 & 981 & Patent W02006039376 \\
\hline & EPSPS 1 & 1415 & NCBI AB209952 \\
\hline & EPSPS 2 & 1367 & Patent WO2004074492 \\
\hline & 2mEPSPS & 1338 & Patent WO2011063411 \\
\hline & bar & 835 & NCBI X05822 \\
\hline & pat & 569 & NCBI GQ497217 \\
\hline & Prsv-cp 1 & 1601 & NCBI F5490192 \\
\hline & Prsv-cp 2 & 1070 & NCBI GZ450610 \\
\hline Miscellaneous & hsp70 & 804 & NCBI AY326434 \\
\hline \multicolumn{2}{|c|}{ Total length of the database used for enrichment } & 52534 & \\
\hline
\end{tabular}

Table 1. List of the structural elements used for the enrichment step. When several variants of the same gene were present, they were identified with a different numeric index (e.g., EPSPS1, EPSPS2).

of reads, standardised in RPKM (reads per kilobase per million mapped reads), obtained for non-GM plants plus five times the calculated standard deviation, giving a probability of differentiation between positive and negative results greater to $99 \%{ }^{27}$. Structural elements were clearly distinguished from each other except for cry gene sequences, as its variants showed similarities in their sequences. However, the highest number of assignations was attributed to the correct cry gene. Non-GM plants were also tested to check for unspecific mappings. With a threshold of 25 reads (standardised in RPKM), no problems were encountered with soybean, cotton or rapeseed. For maize, positive signals were observed with pUbi, pMTL and hsp70, as maize is the donor organism of these structural elements. Some similarities were also identified in maize for the EPSPS1 structural element (GACGAGGAAGCTCATGGCGATGCGGTGATCGAGATGGGTGGCGACG), as this element showed similarity with a 46-bp fragment of the maize genome. Information concerning the donor organism of the structural element and its potential presence in the sample must be taken into account to interpret the results, but the element could also be a target of interest for implementation of the detection system for the identification of plants.

For GMO characterisation, positive reads were assembled to create "blind" contigs to prevent influence from a previously known sequence. This process is important for detecting differences between the announced and 
1. Design of capture probes for each targeted sequence

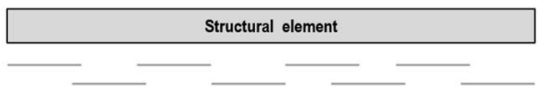

2. Creation of a library of biotinylated capture probes

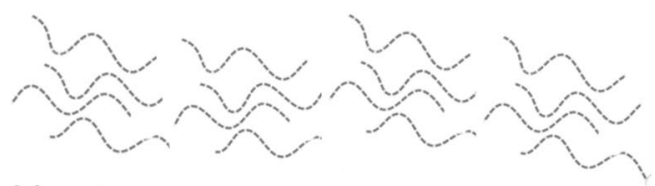

3. Enrichment

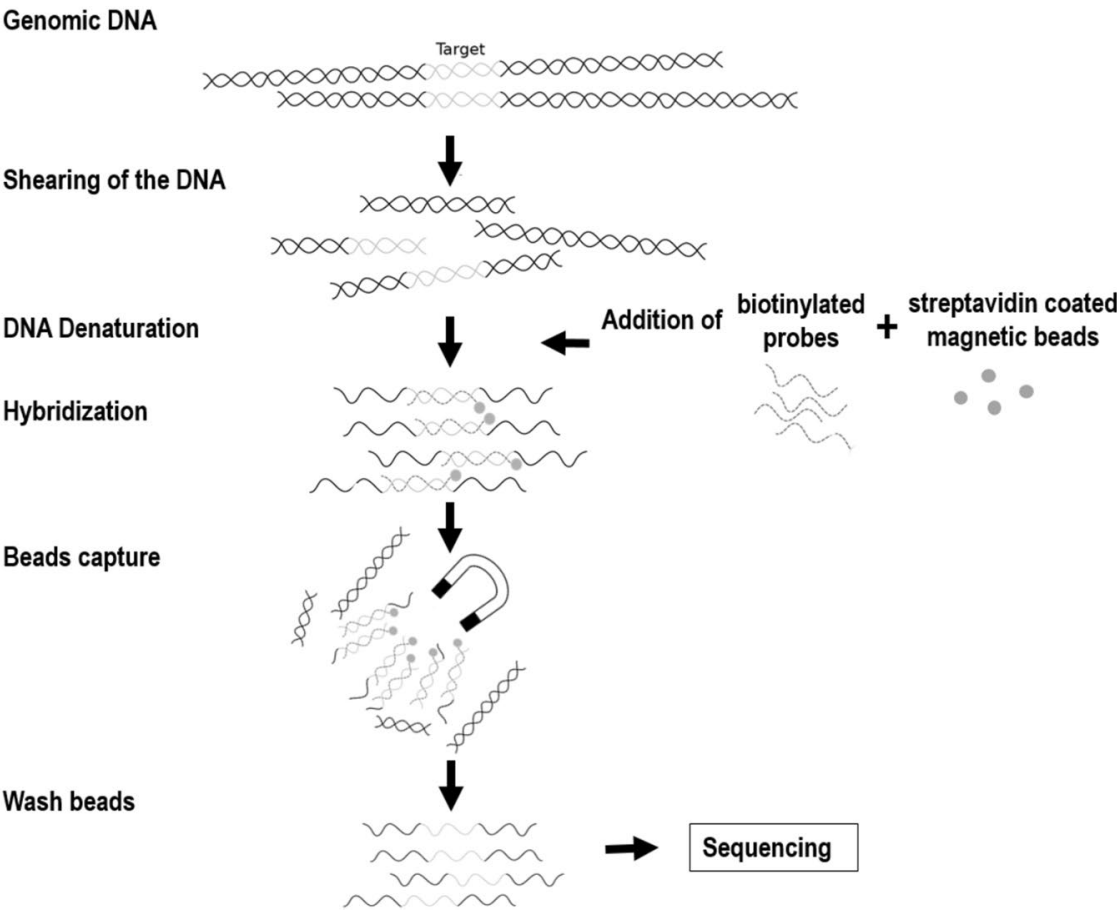

Figure 1. Workflow of the enrichment technology prior to sequencing.

the real sequence of a GMO and to mimic results that could be obtained in the presence of an unknown GMO. Contigs made it possible to partially ( $100 \% 59122$ maize and $10 \% 281 \times 3006$ cotton) or totally ( $10 \%$ GTS-40-3-2 soybean, 100\% GT73 rapeseed, 100\% MS8 rapeseed, 10\% MON89034 maize) reconstruct the sequence of inserts. For $281 \times 3006$ cotton containing three times the pUbi promoter, it was possible to propose contigs for each repetition of the promoter with its respective structural element (Fig. 3), which shows that the method is capable of proposing solutions to help to characterise complex sequences introduced into plants or even mixed GMOs. A sample containing $50 \%$ of A2704 soybean (construct: 35 S promoter - pat gene $-35 \mathrm{~S}$ terminator) and $50 \%$ of LL62 rice (construct: $35 \mathrm{~S}$ promoter - bar gene $-35 \mathrm{~S}$ terminator) was also tested (Fig. 3 ). The bioinformatic pipeline was able to propose a sequence for the inserts introduced in each GM plant. The two sequences were clearly distinguishable even though the sequence of the pat and bar genes showed approximately $60 \%$ similarity when aligned. The sequences of the inserts introduced into A2704 soybean and LL62 rice are not publicly available. Therefore, no comparison between the obtained sequences with the announced sequences was possible. However, the percentage of similarity between known the pat and bar sequences falls into the same range.

Disruptions in the contigs were mainly due to the presence of structural elements that were not originally considered for enrichment and therefore constituted gaps, preventing reassembly of the whole sequence. Adding these elements to future enrichment steps would be an interesting recommendation. A definite advantage of this technology is that fragments caught by the capture probes covered junction regions as well, so it was not only possible to create contigs including junctions between structural elements but also between plant DNA and the GM construct (Figs 3 and 4).

The length of the contigs also depended on the fraction of the GMO in the analysed flour. An example is presented for GTS-40-3-2 soybean (Fig. 3), for which it was possible to assemble contigs even at a percentage as low as $0.1 \%$ of an event, proving that the methodology is very sensitive, as it still succeeded in characterising GMOs 
Assignation and counting of the reads

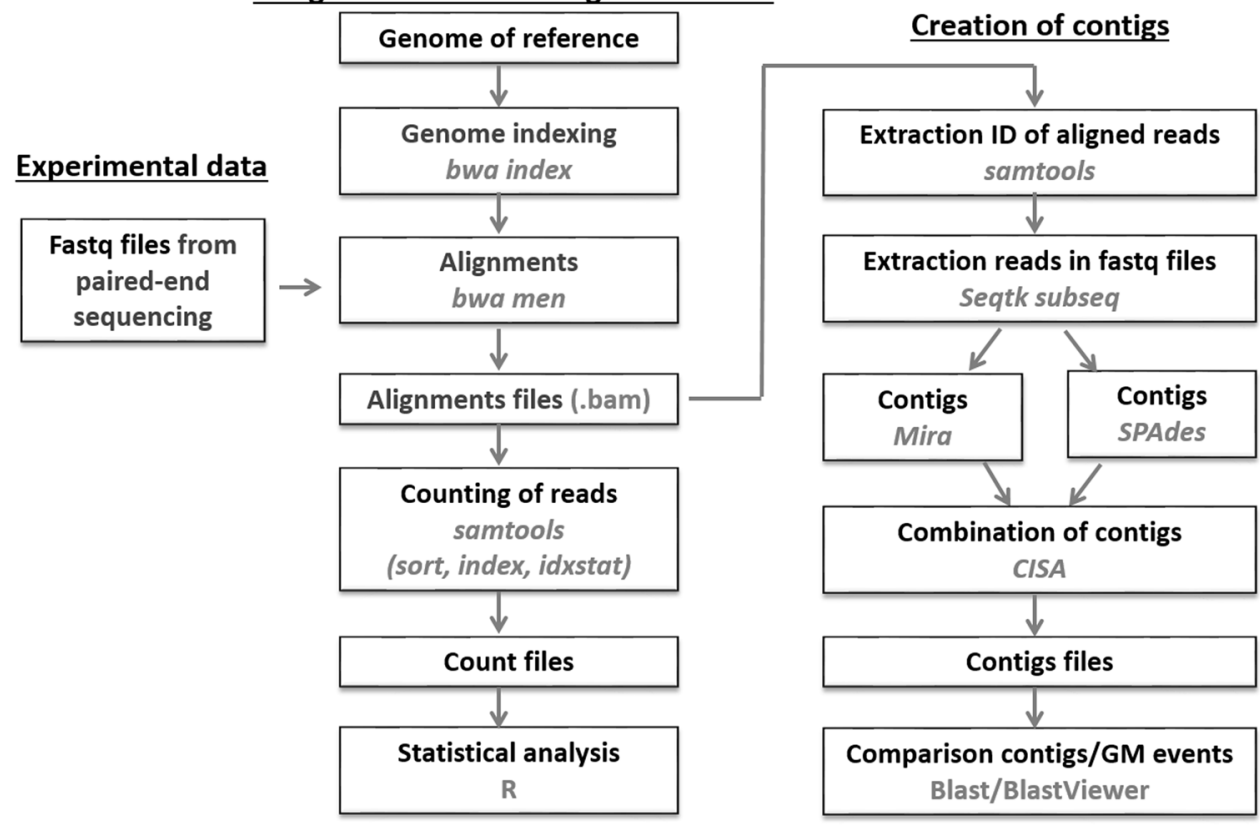

Figure 2. Bioinformatic workflow developed for detecting and identifying GMOs. The bioinformatic packages used are indicated in grey.

\begin{tabular}{|l|l|l|}
\hline Material used & Reference & Provider \\
\hline Rapeseed GT73 $(100 \% \mathrm{GM})$ & AOCS 0304-B & AOCS \\
\hline Rapeseed MS8 $(100 \% \mathrm{GM})$ & AOCS 0306-F2 & AOCS \\
\hline Maize 59122 (10\% GM) & ERM-BF424d & IRMM \\
\hline Cotton $281 \times 3006(10 \% \mathrm{GM})$ & ERM-BF422d & IRMM \\
\hline Maize MON89034 $(100 \% \mathrm{GM})$ & AOCS 0906-E & AOCS \\
\hline Soybean A2704-12 $(100 \% \mathrm{GM})$ & AOCS 0707-B4 & AOCS \\
\hline Rice LL62 $(100 \%$ GM $)$ & AOCS 0306-I4 & AOCS \\
\hline Soybean GTS 40-3-2 $(0.1 \%$ GM) & ERM-BF410bk & IRMM \\
\hline Soybean GTS 40-3-2 $(1 \%$ GM) & ERM-BF410dk & IRMM \\
\hline Soybean GTS 40-3-2 $(10 \%$ GM) & ERM-BF410gk & IRMM \\
\hline Maize $(0 \%$ GM $)$ & Commercial organic maize & (Ekibio, Peaugres, France) \\
\hline Soybean $(0 \%$ GM) & Commercial organic soybean & (Ekibio, Peaugres, France) \\
\hline
\end{tabular}

Table 2. Origin of the samples used for analysis by NGS.

at low percentages. For GTS-40-3-2, at a level as low as $1 \%$ of GM, it was possible to recreate the transgenic construct and determine the left border (plant-GM construct junction) and the rearranged sequence as described by Windels et al. ${ }^{28}$ on the right side. This rearrangement corresponds to a portion of the EPSPS gene and a part of the plasmid vector used for transformation. The contig for GTS40-3-2 soybean at 1\% was somewhat shorter than the contig obtained for GTS40-3-2 at 10\%. At $0.1 \%$, it was possible to create two contigs, with one of them covering the left junction (plant - DNA construct). The lower number of reads available in this last case made it impossible to reconstruct the whole sequence of the transgenic cassette.

DNA enrichment has a cost of 300 euros/sample and sequencing adds additional 300 euros/sample. This price is high for an analysis in the field of agrofood products, but since the first experiments, conducted 3 years ago, the estimated cost of the approach has already been halved. If the time required to perform enrichment ( 2 days), sequence the libraries ( 2 days) and complete the bioinformatics analysis ( 3 hours/sample) is reasonable for a routine analysis, access to a sequencing machine - if outsourced - generally takes at least one month and remains a very limiting factor when a fast answer is needed. Therefore, the use of affordable machines (e.g., minion, Oxford Nanopore technologies, Oxford, UK) must be tested in future approaches ${ }^{29}$.

The sequencing approach can be used: (i) alone, as a new detection and characterisation technique that has a good coverage because of the large number of structural elements tested; (ii) as a complement to real-time PCR to characterise the GM construct(s) or event(s) initially detected by real-time PCR tests; and (iii) prior to the development of an event-specific real-time PCR test because of the characterisation of the GM insert and its border regions. 
GT73 rapeseed (100\% GM)

\begin{tabular}{|l|l|l|l|l|l|l|l|}
\hline pFMV & ctp1 & gox & tE9 & pFMV & ctp2 & cp4EPSPS & tE9 \\
\hline
\end{tabular}

MS8 rapeseed (100\% GM)

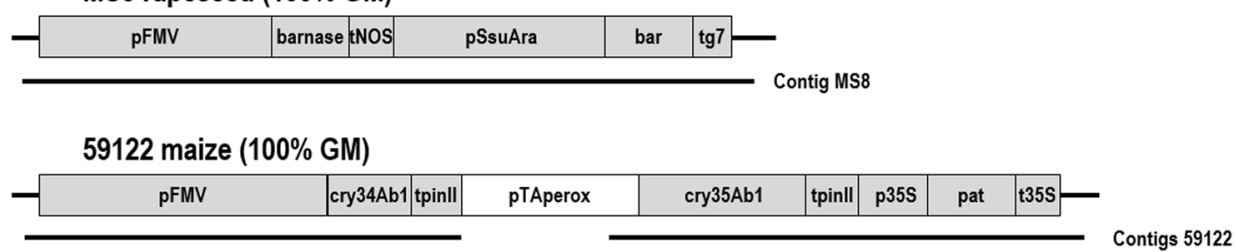

$281 \times 3006$ cotton $(10 \% \mathrm{GM})$

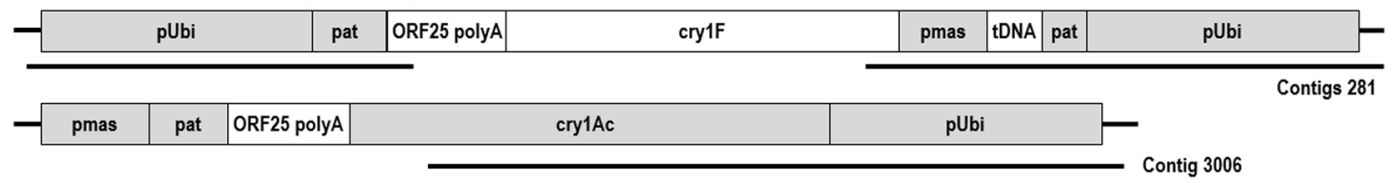

MON89034 maize (100\% GM)

\begin{tabular}{|c|c|c|c|c|c|c|c|c|c|}
\hline p35s & L-cab & I-rAct & cry1A105 & \begin{tabular}{|l|} 
tahsp17 \\
\end{tabular} & pFMV & hsp70 & Ctp-SSU & cry2Ab2 & tNOS \\
\hline
\end{tabular}

Mix A2704 soybean ( $100 \% \mathrm{GM})$ and LL62 rice (100\% GM)

\begin{tabular}{|c|c|c|}
\hline p35s & pat & t35 \\
\hline & $=500 \mathrm{bp}$ \\
\end{tabular}

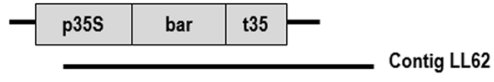

Figure 3. Detection and characterisation of GMOs by NGS. The structures of the inserts of seven GMOs are presented. The $281 \times 3006$ cotton has two GM inserts. The mixed sample contains $50 \%$ A2704 soybean and $50 \%$ LL62 rice. The structural elements in grey were present in the database used for enrichment and were detected by NGS. The reads associated with these structural elements were used to create contigs. Only larger contigs covering several structural elements are shown here. Larger structural elements not covered by the capture probes created gaps, making it impossible to reconstruct the entire sequence of the transgenic cassette. Junction regions covering the plant and transgenic insert were also obtained.

\section{A. Expected sequences}

\begin{tabular}{|c|c|c|c|c|}
\hline p35S & ctp4 & cp4EPSPS & tNOS & Announced GTS 40-3-2 soybean \\
\hline p35S & ctp4 & cp4EPSPS & tNOS cp4EPSPS rearrangement & $\begin{array}{l}\text { Rearrangement observed by } \\
\text { Windels et al., 2001 }\end{array}$ \\
\hline
\end{tabular}

\section{B. Contigs obtained after assembly of the reads}

\begin{tabular}{|c|c|c|c|c|}
\hline p35s & ctp4 & cp4EPSPS & tNOS 'cp4EPSPS' rearrangement & GTS $40-3-2$ soybean ( $10 \%$ GM) \\
\hline p35s & ctp4 & cp4EPSPS & tNOS 'cp4EPSPS' rearrangement - & GTS $40-3-2$ soybean ( $1 \%$ GM) \\
\hline p35s & ctp4 & cp4EPSPS & tNOS 'cp4EPSPS' rearrangement - & GTS $40-3-2$ soybean $(0.1 \%$ GM) \\
\hline
\end{tabular}

Figure 4. Sequence of GTS 40-3-2 soybean and alignments of the contigs obtained in this research. The structural elements in grey shown in the database were used for enrichment and were detected by NGS. (A) Expected sequences of the GTS-40-3-2 soybean, as announced by Monsanto and as described by Windels et al. ${ }^{28}$. Additional sequence corresponds to a duplication of part of the EPSPS gene and an unknown rearranged sequence. (B) Positions of the contigs created for the samples containing GTS- $40-3-2$ soybean at $10 \%, 1 \%$ and $0.1 \%$. 


\begin{tabular}{|l|c|c|c|c|}
\hline Reads & $\begin{array}{c}\text { Soybean } \\
\text { 0\% GM }\end{array}$ & $\begin{array}{c}\text { Soybean } \\
\text { GTS 40-3-2 } \\
\mathbf{0 . 1 \%} \text { GM }\end{array}$ & $\begin{array}{c}\text { Soybean } \\
\text { GTS 40-3-2 } \\
\text { 1\% GM }\end{array}$ & $\begin{array}{c}\text { Soybean } \\
\text { GTS 40-3-2 } \\
\mathbf{1 0 \%} \text { GM }\end{array}$ \\
\hline Number of reads & 2103154 & 2098020 & 2226922 & 1961322 \\
\hline Number of reads aligned & 211 & 1423 & 11023 & 74739 \\
\hline \% of reads aligned & 0.01 & 0.07 & 0.49 & 3.81 \\
\hline Reads aligned with p35S & 0 & 128 & 1275 & 10504 \\
\hline Reads aligned with tNOS & 4 & 142 & 1438 & 8743 \\
\hline Reads aligned with EPSPS & 28 & 824 & 8107 & 55316 \\
\hline
\end{tabular}

Table 3. Reads obtained in soybean flour containing $0.1 \%, 1 \%$ and $10 \%$ GTS $40-3-2$ soybean (results obtained with reads of $75 \mathrm{bp}$; the number of reads was not normalised to the length of the structural element considered here). The values presented in the table are the results of a single analysis.

Approaches to GMO detection using NGS have been proposed before, but this is the first time that such a methodology (i) enables the detection of GMOs at low levels, (ii) can be used on products containing several plant species, (iii) focuses on a large panel of screening elements, and (iv) makes it possible to partially or completely reconstruct a GMO, thereby providing a mechanism to detect unknown events. In the case of a laboratory equipped with NGS technology, this methodology could also be applied in a time frame that is more suitable for routine analysis.

Moreover, this is the first step towards a more informative analysis, as the enrichment can be extended to sequences corresponding to additional structural elements, plant species, allergens and contaminants. Specific sequences for these elements can be added to the database for the design of capture probes, leading to a technology not only focused on GMO detection but also extendable to the determination of other interesting food and feed product features. The strategy described in this study is only valid for GMOs obtained through classical recombinant DNA technology that give rise to transgene constructs. This study is not aimed at gene editing techniques (e.g., CRISPR/Cas9).

\section{Methods}

Samples. The certified transgenic reference materials (CRMs) were obtained from the Institute for Reference Materials and Measurements (JRC, Geel, Belgium) and the American Oil Chemists' Society (AOCS, Urbana, Illinois, USA). Commercial organic grains were collected for non-GM plant species. Tests performed using realtime PCR ${ }^{30,31}$ confirmed the absence of GM material from commercial organic grains. The origin of the material is presented in the supplementary material (Table 2).

The samples were considered individually for sequencing (with the exception of the 50\% LL62 rice/50\% A2704-12 soybean mix), and some of the samples (maize 0\% GM, soybean 0\% GM, maize MON89034 100\%) were repeated to observe background noise.

DNA extraction. Genomic DNA was extracted and purified from all samples following the CTAB-based method described in Annex A.3.1 of the ISO 21571:2005 international standard ${ }^{32}$. The quality of DNA extracted from samples was estimated using a Nanodrop ND-1000 spectrophotometer (Nanodrop Technologies, Wilmington, DE). DNA samples were quantified by Picogreen (Quant-iT ${ }^{\mathrm{TM}}$ PicoGreen $^{\mathrm{TM}}$ dsDNA Assay Kit, Invitrogen, Carlsbad, CA); $3 \mu \mathrm{g}$ of DNA was used for library preparation.

Next generation sequencing. DNA was sheared on a Picoruptor (Diagenode, Liège, Belgium) to produce fragments of $\sim 150-200 \mathrm{bp}$. The SureSelect XT Target Enrichment system (Agilent technologies) was used to capture sequences of interest prior to sequencing. The design includes 458 enrichment probes. The sequences of the probes are available in supplementary material (Table S1). Via the online tool "Suredesign" on the Agilent Technologies website and through the option "collaboration space" with reference to design ID 3045501, probes were ordered from Agilent. No degeneracy was introduced in the sequences of the probes. Sequencing was performed on an Illumina MiSeq instrument with MiSeq Reagent Kit v3 $(2 \times 75 \mathrm{bp})$ at the GIGA Genomics platform at the University of Liège.

The pipeline for analysing results was perfected, as shown in Fig. 2, by the use of free access programs (with their default settings): bwa mem version $0.7 .16-\mathrm{r} 1180^{33}$, Samtools version $0.1 .19-96 \mathrm{~b} 5 \mathrm{f} 2294 \mathrm{a}^{34}$, R ggplot version 2_2.2.1 ${ }^{35}$, Seqtk version $1.2^{36}$, Velvet version $1.2 .10^{37}$, Mira version $4.0 .2^{38}$, Spades version $3.12^{39}$, CISA version $1.3^{40}$ and Blast version $2.7 .1+{ }^{41,42}$. The commands calling the different packages and an example (manifest file) are given in Supplementary Material S2.

The assembled contigs were compared to the sequences of the inserts introduced in plants: GTS 40-3-2 soybean (Windels et al., 2001), GT73 rapeseed (patent US 6248876), MS8 (structure of the plasmid pTHW101 as described in notification C/BE/96/01), 59122 maize (NCBI accession HW057200), $281 \times 3006$ cotton (patents EP2333082 and EP2862934) and MON89034 maize (NCBI accession FV532179).

Received: 20 August 2018; Accepted: 20 September 2019;

Published online: 30 October 2019 


\section{References}

1. Michael, T. P. \& Jackson, S. The first 50 plant genomes. Plant Genome 6 (2013).

2. Kovalic, D. et al. The use of next generation sequencing and junction sequence analysis bioinformatics to achieve molecular characterization of crops improved through modern biotechnology. Plant Genome 5, 149-163 (2012).

3. Wahler, D. et al. Next-generation sequencing as a tool for detailed molecular characterisation of genomic insertions and flanking regions in genetically modified plants: a pilot study using a rice event unauthorised in the EU. Food Anal. Meth. 6, 1718-1727 (2013).

4. Yang, L. et al. Characterization of GM events by insert knowledge adapted re-sequencing approaches. Sci. Rep. 3 (2013).

5. Liang, C. et al. Detecting authorized and unauthorized genetically modified organisms containing vip3A by real-time PCR and nextgeneration sequencing. Anal. Bioanal. Chem. 406, 2603-2611 (2014).

6. Holst-Jensen, A. et al. Application of whole genome shotgun sequencing for detection and characterization of genetically modified organisms and derived products. Anal. Bioanal. Chem. 408, 4595-4614 (2016).

7. Willems, S. et al. Statistical framework for detection of genetically modified organisms based on Next Generation Sequencing. Food Chem. 192, 788-798 (2016).

8. Schmutz, J. et al. Genome sequence of the palaeopolyploid soybean. Nature 463, 178-183 (2010).

9. Shi, X. \& Ling, H. Q. Current advances in genome sequencing of common wheat and its ancestral species. Crop J. 6, 15-21 (2018).

10. Tengs, T. et al. Microarray-based method for detection of unknown genetic modifications. BMC biotechnol. 7, 91 (2007).

11. Tengs, T. et al. Characterization of unknown genetic modifications using high throughput sequencing and computational subtraction. BMC biotechnol. 9, 87 (2009).

12. Tengs, T. et al. Non-prejudiced detection and characterization of genetic modifications. Food Anal. Methods 3, 120-128 (2010).

13. Arulandhu, A. J. et al. NGS-based amplicon sequencing approach; towards a new era in GMO screening and detection. Food Control 93, 201-210 (2018).

14. Fraiture, M. A. et al. Validation of a sensitive DNA walking strategy to characterise unauthorised GMOs using model food matrices mimicking common rice products. Food Chem. 173, 1259-1265 (2015).

15. Košir, A. B. et al. ALF: a strategy for identification of unauthorized GMOs in complex mixtures by a GW-NGS method and dedicated bioinformatics analysis. Sci. Rep. 8, 17645 (2018).

16. Fraiture, M. A. et al. Development and validation of an integrated DNA walking strategy to detect GMO expressing cry genes. BMC biotechnol. 18, 40 (2018).

17. Arulandhu, A. J. et al. DNA enrichment approaches to identify unauthorised genetically modified organisms (GMOs). Anal. Bioanal. Chem. 408, 4575-4593 (2016).

18. Block, A. et al. The GMOseek matrix: a decision support tool for optimizing the detection of genetically modified plants. BMC Bioinformatics 14, 256 (2013).

19. Debode, F. Développement de méthodologies pour la détection des plantes génétiquement modifiées. Phd Thesis, AGRO, UCL, 367/2017, 391 p., http://hdl.handle.net/2078.1/186329 (2017).

20. Angenon, G. et al. Antibiotic resistance markers for plant transformation. In Plant molecular biology manual, Springer, Dordrecht, 125-137 (1994).

21. Sulonen, A. M. et al. Comparison of solution-based exome capture methods for next generation sequencing. Genome Biol. 12, 94 (2011).

22. Teer, J. K. Systematic comparison of three genomic enrichment methods for massively parallel DNA sequencing. Genome Res. 20, 1420-1431 (2010).

23. Bodi, K. et al. Comparison of commercially available target enrichment methods for next-generation sequencing. J. Biomol. Tech. 24, 73 (2013)

24. Meienberg, J. et al. New insights into the performance of human whole-exome capture platforms. Nucleic Acids Res. 43, 1-14 (2015).

25. Chilamakuri, C. S. R. et al. Performance comparison of four exome capture systems for deep sequencing. BMC genomics $\mathbf{1 5}, 449$ (2014).

26. García-García, G. et al. Assessment of the latest NGS enrichment capture methods in clinical context. Sci. Rep. 6, 20948 (2016).

27. IUPAC Compendium of Chemical Terminology, 2nd ed. (Compiled by McNaught, A. D. \& Wilkinson A.). Blackwell Scientific Publications, Oxford., 464 pages. ISBN 0-9678550-9-8 (1997).

28. Windels, P. et al. Characterisation of the Roundup Ready soybean insert. Eur. Food Res. Technol. 213, 107-112 (2001).

29. Fraiture, M. A. et al. Nanopore sequencing technology: a new route for the fast detection of unauthorized GMO. Sci. Rep. 8, 7903 (2018).

30. Debode, F. et al. Development of 10 new screening PCR assays for GMO detection targeting promoters (pFMV, pNOS, pSSuAra, pTA29, pUbi, pRice actin) and terminators (t35S, tE9, tOCS, tg7). Eur. Food Res. Technol. 236, 659-669 (2013).

31. Debode, F. et al. Development of PCR screening assays focused on gene-coding sequences for GMO detection. Biotechnol. Agron. Soc. Environ. 22, 230-241 (2018).

32. ISO 21571. Foodstuffs. Methods of analysis for the detection of genetically modified organisms and derived products. Nucleic acid extraction. International Organization for Standardization, Geneva (2005).

33. Li, H. \& Durbin, R. Fast and accurate short read alignment with Burrows-Wheeler transform. Bioinformatics 25, 1754-1760 (2009).

34. Li, H. et al. The sequence alignment/map format and SAMtools. Bioinformatics 25, 2078-2079 (2009).

35. Wickham, H. ggplot2: elegant graphics for data analysis. J. Stat. Softw. 35, 65-88 (2010).

36. Li, H. Seqtk Toolkit for processing sequences in FASTA/Q formats, https://github.com/lh3/Seqtk (2012).

37. Zerbino, D. R. \& Birney, E. Velvet: algorithms for de novo short read assembly using de Bruijn graphs. Genome Res. 18, 821-829 (2008).

38. Chevreux, B. et al. Genome Sequence Assembly Using Trace Signals and Additional Sequence Information. Computer Science and Biology. In: Proceedings of the German Conference on Bioinformatics, 45-56 (1999).

39. Bankevich, A. et al. SPAdes: a new genome assembly algorithm and its applications to single-cell sequencing. J. Comput. Biol. 19, 455-477 (2012).

40. Lin, S. H. \& Liao, Y. C. CISA: contig integrator for sequence assembly of bacterial genomes. PloS one 8, e60843 (2013).

41. Morgulis, A. et al. Database indexing for production MegaBLAST searches. Bioinformatics 24, 1757-1764 (2008).

42. Camacho, C. et al. BLAST+: architecture and applications. BMC bioinformatics 10, 421 (2009).

\section{Acknowledgements}

The first strategy of this research using NimbleGen technology was designed within a Belgian research project (Convention RF 11/6242 UGMMONITOR) financed by the Belgian Federal Public Service for Public Health, Food Chain Safety and Environment. The second strategy, using SureSelect technology, was financed by CRA-W in the framework of the NGS project (Moerman funds). We thank Cécile Ancion, Denis Roulez, Gaëlle Antoine and Eric Janssen from the GMO team of CRA-W for their help in the preparation of DNA. We thank the GIGA Genomics Platform for technical assistance with NGS data generation and analysis. 


\section{Author contributions}

F.D. designed the experiments and strategies; J.H. completed the bioinformatic pipeline for the analysis of the results with advice from B.C., W.C. and M.H.; B.C., L.K. and W.C. supervised the sequencing; G.B. supervised the work and funding; F.D. wrote the manuscript with comments from all the authors.

\section{Competing interests}

The authors declare no competing interests.

\section{Additional information}

Supplementary information is available for this paper at https://doi.org/10.1038/s41598-019-51668-x.

Correspondence and requests for materials should be addressed to F.D.

Reprints and permissions information is available at www.nature.com/reprints.

Publisher's note Springer Nature remains neutral with regard to jurisdictional claims in published maps and institutional affiliations.

(c) (i) Open Access This article is licensed under a Creative Commons Attribution 4.0 International License, which permits use, sharing, adaptation, distribution and reproduction in any medium or format, as long as you give appropriate credit to the original author(s) and the source, provide a link to the Creative Commons license, and indicate if changes were made. The images or other third party material in this article are included in the article's Creative Commons license, unless indicated otherwise in a credit line to the material. If material is not included in the article's Creative Commons license and your intended use is not permitted by statutory regulation or exceeds the permitted use, you will need to obtain permission directly from the copyright holder. To view a copy of this license, visit http://creativecommons.org/licenses/by/4.0/.

(c) The Author(s) 2019 